\section{RECENT RESULTS FROM WIMP-SEARCH ANALYSIS OF CDMS-II DATA}

2. AHMED* for the CDMS-II collaboration

Division of Mathematics, Physics and Astronomy, Califorria Institute of Technology. Pasadena, CA 91125, USA

*E-mail: zahmed@caltech.ed

The Cryogenic Dark Matter Search (CDMS-II) experiment, at Soudan Underground Laboratory, used germanium low-temperature particle detectors to search for Weakly Interacting Massive Particles (WIMPs), characterized by elastic nuclear scattering. We report results from the analysis of final data taken with the CDMS-II apparatus. Two events were observed in the signal region. Based on our background estimate, the probability of observing two or more background events is $23 \%$. Combined with previous CDMS-II data, this results in an upper limit on the WIMP-nucleon spin-independen interaction cross-section of $3.8 \times 10^{-44} \mathrm{~cm}^{2}$ at $90 \%$ CL for a $70 \mathrm{GeV} / \mathrm{c}^{2}$ WIMP. CDMS-II ended operations in March 2009, to be upgraded to SuperCDMS with detectors that are 2.5 times more massive and have improved background rejection. The first set of such detectors has been deployed at Soudan and is taking data.

Keywords: Dark Matter; WIMPs; CDMS.

\section{Introduction}

Cosmological evidence [1] indicates that only $\sim 4 \%$ of energy density of the universe is comprised of baryons, although more than $25 \%$ is contained in matter. The missing matter or dark matter is likely not only non-baryonic, but non-relativistic at the time of structure formation. One candidate for cold dark matter is Weakly Interacting Massive Particles (WIMPs) [2], well-motivated from a cosmological thermal relic framework and independently from proposed extensions of the Standard Model such as Supersymmetry [3-5].

WIMPs are expected to have scattering cross-sections on the order of the weak scale, and masses around $\sim 100 \mathrm{GeV} / \mathrm{c}^{2}[6]$. As dark matter particles they would constitute diffuse halos around galaxies with isothermal velocities [7]. Terrestrially, they would appear to originate from the direction opposite to that of the Solar System's motion through the Milky Way with an average velocity of $270 \mathrm{~km} / \mathrm{s}$. WIMPs would elastically scatter of nuclei in particle detectors thus producing a roughly exponential energy deposition spectrum, averaged around few tens of $\mathrm{keV}$, with an interaction rate of $<0.1$ events $/ \mathrm{kg} /$ day $[8,9]$.
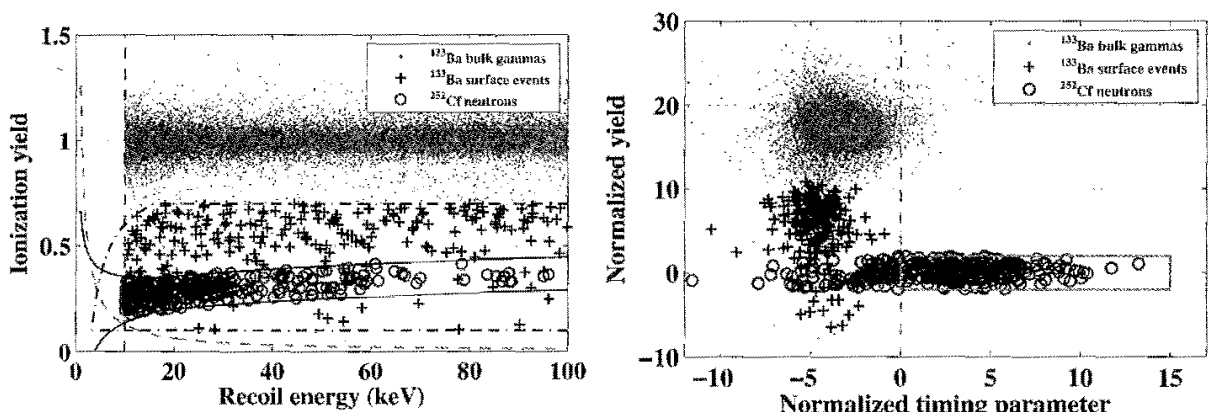

Fig. 1. Background discrimination for a typical Ge detector demonstrated using in situ calibration sources. Bulk electron recoils (red dots) and low-yield surface events (black - t) from a ${ }^{133^{3} \mathrm{Ba}}$ source and neutron-induced nuclear recoil events (blue of from a ${ }^{252} \mathrm{Cf}$ are marked. [Left] Ionization yield and neutron-induced nuclear recil enents (blue verus resto dashed magnta line indicate the recoil energy analysis thrashol.. Jhe ragion enclosed in the dot dashed lines indicates calibration events used to set the surface-event rejection cut. [Right] Normalized ionization yield (number of standard deviations from mean of nuclear recoil band) versus normalized timing parameter (timing relative to acceptance region) is shown for the same data. Events to the right of the vertical red dashed line pess the surface-event rejection cut for this detector. The solid red box is the WIMP signal region. Reproduced from Ref. [10].

\section{CDMS-II Experiment}

The Cryogenic Dark Matter Search (CDMS-II) experiment consisted of an array of 19 germanium $(250 \mathrm{~g})$ and 11 silicon $(100 \mathrm{~g})$ particle detectors operated at cryogenic temperature $(\sim 50 \mathrm{mK})[11,12]$. Each detector was a cylindrical disk, $7.6 \mathrm{~cm}$ in diameter and $1 \mathrm{~cm}$ thick. The detectors are grouped into five towers, each tower containing six detectors. Detectors are identified by their tower number (T1-T5) and their position within that tower (Z1-Z6). Particle interactions in a detector generated ionization as well as athermal phonons. An electric field across the detector separated the resulting electrons and holes which were collected on electrodes patterned on the flat faces, producing an ionization energy measurement. Phonons were collected in four superconducting thin-film absorber circuits and the energy was read out using tungsten transition-edge sensors (TESs) coupled with superconducting quantum interference devices (SQUIDs). A direct line of sight between adjacent detectors in a tower allows identification of events scattering between detectors.

The ionization yield, or the ratio of charge to phonon energy depositions, provided the primary discrimination between electron recoils and nuclear recoils to better than $10^{-4}$ misidentification rate. For events within $10 \mu \mathrm{m}$ of a detector surface, charge collection was suppressed and ionization yield was reduced. Additional discrimination was obtained from the promptness of phonon pulses; surface events had faster pulses than bulk events. Combining ionization yield and phonon timing, bulk electron recoils were rejected to better than $10^{-6}$ misidentification rate and surface electron recoils to better than $10^{-2}$. This is illustrated in Fig. 1 using 


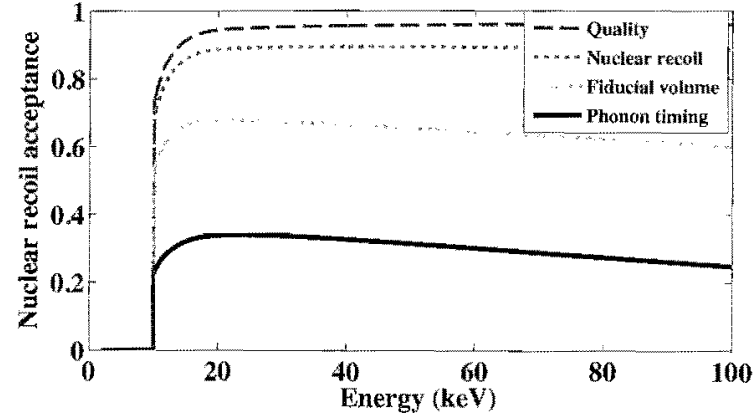

Fig. 2. Signal criteria efficiency versus recoil energy. Each line represents the cumulative effect of the criterion combined with the ones above it.

\section{calibration data}

In order to suppress ambient photons and radiogenic neutron rates, the entire experimental apparatus was surrounded by layers of lead and polyethylene. Finally, the experiment was situated at the Soudan Underground Mine at a depth of 2090 m.w.e. to suppress muon flux. An active plastic scintillator veto further tagged remaining incident muons which interacted in the apparatus to generate cosmogenic neutrons [11].

\section{Results}

We report results from the final WIMP-search data acquired between July 2007 and September 2008. At regular intervals during data acquisition, as well as collectively afterwards, detector performance was characterized by automated checks of detector neutralization (required for full ionization collection) and Kolmogorov-Sminrov tests on various parameter distributions including charge and phonon pulse characteristics. All 30 detectors (Ge and Si) were used to identify particle interactions, but only optimally performing Ge detectors were used to search for WIMP scatters, leading to $612 \mathrm{~kg}$-days of net WIMP-search exposure.

To prevent bias, the definition of physics cuts, calculation of their efficiencies, and characterization of detector response was done only using calibration data from ${ }^{133} \mathrm{Ba}$ and ${ }^{252} \mathrm{Cf}$ sources, or events in WIMP-search data outside the signal region. $356 \mathrm{keV} \gamma$-rays from ${ }^{133} \mathrm{Ba}$ were used to calibrate the ionization and phonon energy scales of the detectors. The validity and linearity of this calibration were verified at WIMP-scatter energies of interest using $10.36 \mathrm{keV}$ x-rays from neutron activation of ${ }^{70} \mathrm{Ge}$. Calibration data also provided sample surface events and nuclear recoils (Fig. 2) to tune the phonon timing based surface-event rejection cut for maximum sensitivity to a $60 \mathrm{GeV} / \mathrm{c}^{2}$ WIMP.

WIMP candidates were defined as events with recoil energies between $10 \mathrm{keV}$ and $100 \mathrm{keV}$, within the detector fiducial volume, anti-coincident with muon veto activity, having interacted only in a single detector in the apparatus, within $2 \sigma$ of the
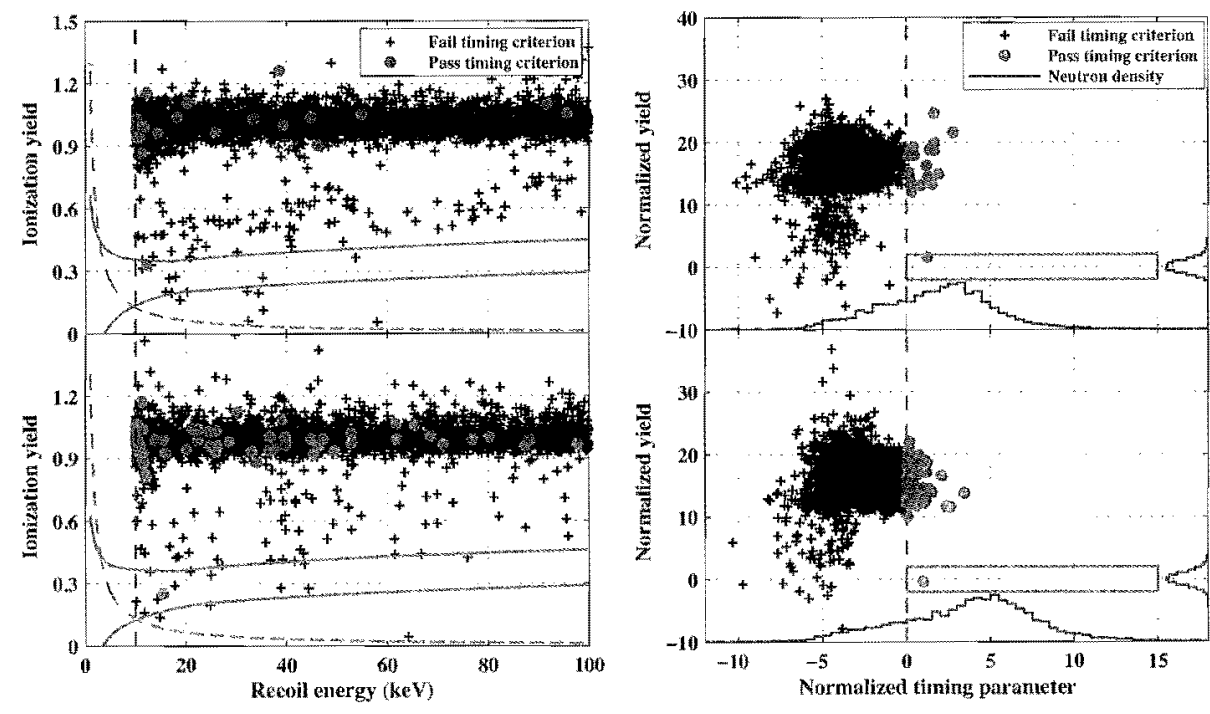

Fig. 3. [Left] Ionization yield versus recoil energy for events passing all cuts, excluding yield and timing. The top (bottom) plot shows events for detector T1Z5(T3Z4). The solid red lines indicate the $2 \sigma$ electron and nuclear recoil bands. The vertical dashed line represents the recoil energy threshold and the sloping magenta dashed line is the ionization threshold. Events that pass the timing cut are shown with round markers. The candidate events are the round markers inside the nuclear-recoil bands. [Right] Normalized ionization yield (number of standard deviations from mean of muclear recoil band) versus normalized timing parameter (timing relative to acceptance region) for events passing all cuts, excluding yield and timing. The top (bottom) plot shows events for detector T1Z5(T3Z4). Events that pass the phonon timing cut are shown with round markers The solid red box indicates the signal region for that detector. The candidate events are the round markers inside the signal regions. Also shown for reference are normalized timing parameter and normalized ionization yield histograms for calibration neutrons. Reproduced from Ref. [10].

mean ionization yield of calibration-neutron events and having failed a surface event cut based on phonon pulse timing. The efficiency of these criteria were measured as a function of energy and are plotted in Fig. 2. The WIMP-spectrum-averaged equivalent exposure for a WIMP of mass $60 \mathrm{GeV} / \mathrm{c}^{2}$ was $194 \mathrm{~kg}$-days.

Prior to unblinding. we also estimated the expected contribution of various background sources. The cosmogenic neutron background was estimated to be $0.04_{-0.03}^{+0.04}($ stat. $)$ by Monte Carlo simulations of muon-induced particle showers and subsequent neutron production. The radiogenic background was estimated to be between 0.03 and 0.06 events based on counting of shielding and detector material samples. The expected background contribution from surface events was $0.6 \pm 0.1$ (stat.), estimated using pass-fail ratios of the surface-event cut, measured on calibration surface events and WIMP-search events outside the signal region.

With the analysis finalized, the blind signal region was unmasked on November 5,2009 . We observed two events in the WIMP-acceptance region at recoil energies of $12.3 \mathrm{keV}$ and $15.5 \mathrm{keV}$. These are marked in Fig. 3. 

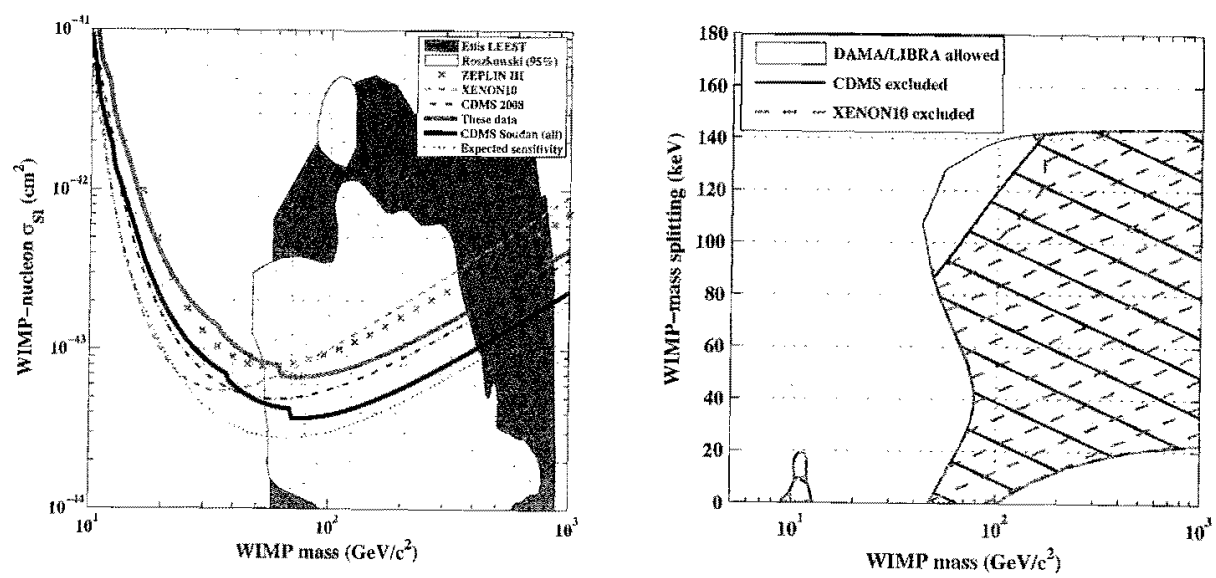

Fig. 4. [Left] 90\% C.L. upper limits on the WIMP-nucleon spin-independent cross section as a function of WIMP mass. The red (upper) solid line shows the limit obtained from the exposure analyzed in this work. The solid black line shows the combined limit for the full data set recorded at Soudan. The dotted line indicates the expected sensitivity for this exposure based on our estimated background combined with the observed sensitivity of past Soudan data. Prior results from CDMS [12]: LDELWEISS III [13], XENON10 [14], and ZEPLIN III [15] are shown for comparison. The shaded regions indicate allowed parameter space calculated from certain Minimal Supersymmetric Models $[16,17][$ Right] The shaded blue region represents WIMP masses and mass splittings for which there exists a cross section compatible with the DAMA/LIBRA [18] modulation spectrum at $90 \%$ C. L. under the inelastic dark matter interpretation [19]. Excluded regions for CDMS II (solid-black hatched) and XENON10 [20] (red-dashed hatched) were calculated in this work using the Optimum Interval Nethod. Reproduced from Ref. [10]

The candidate events occurred in periods of ideal experimental performance, separated in time by several months, and in different detectors in the apparatus. However, a detailed study revealed degraded surface event rejection for a small fraction of events with ionization energy below $\sim 6 \mathrm{keV}$, due to misconstructed event timing. Accounting for this effect, the surface background estimate stood revised to $0.8 \pm 0.1($ stat. $) \pm 0.2$ (syst.). Combining this with the estimated neutron background, the probability to observe two or more background events is $23 \%$. We studied the proximity of the candidates to the surface-event rejection threshold by varying the timing cut threshold of the analysis. By reducing the expected surface event background to 0.4 events, both events are removed at a loss of $28 \%$ in WIMP-exposure No additional events would be added by increasing the expected surface event background to 1.7 events.

Based on these and previous CDMS data, we set a combined limit on spinindependent WIMP-nucleon interactions of $3.8 \times 10^{-44} \mathrm{~cm}^{2}$ at $90 \% \mathrm{CL}$ for $70 \mathrm{GeV} / \mathrm{c}^{2}$ WIMP, [10], based on standard galactic halo assumptions [21]. We use the Optimum Interval Method [22], with no background subtraction. The left pane of Fig. 4 shows this limit plotted along with other recent results and favored parameter space under various theoretical models.
These data were also analyzed under the hypothesis of WIMP inelastic scattering [19], which was proposed to explain the DAMA/LIBRA data [18]. We computed DAMA/LIBRA regions allowed at the $90 \%$ C.L. following the $\chi^{2}$ goodness-of-fit technique described in [23], without including channeling effects [24]. Limits from our data and that of XENON10 [20] were computed using the Optimum Interval Method [22]. Regions excluded by CDMS and XENON10 were defined by demanding the $90 \%$ C.L. upper limit to completely rule out the DAMA/LIBRA allowed cross section intervals for allowed WIMP masses and mass splittings. The results are shown in the right pane of Fig. 4.

\section{Current Status}

CDMS-II ended operations in March 2009, and is being upgraded to SuperCDMS Soudan. CDMS-II detectors are being replaced with new ones, 2.5 times more massive than the old ones and with phonon sensors redesigned for better surface event rejection. The first tower of new detectors has already been deployed and is taking data at Soudan. By Summer 2010, 15kg of Ge detectors will be deployed in SuperCDMS with the goal of probing WIMP-nucleon cross-sections of $5 \times 10^{-45} \mathrm{~cm}^{2}[25]$.

\section{Acknowledgments}

The CDMS collaboration gratefully acknowledges the contributions of numerous engineers and technicians; we would like to especially thank Jim Beaty, Bruce Hines, Larry Novak, Richard Schmitt and Astrid Tomada. This work is supported in part by the National Science Foundation (Grant Nos. AST-9978911, PHY-0542066, PHY-0503729, PHY-0503629, PHY-0503641, PHY-0504224, PHY0705052, PHY-0801708, PHY-0801712, PHY 0802575 and PHY-0855525), by the Department of Energy (Contracts DE-AC03-76SF00098, DE-FG02-91ER40688, DE-FG02-92ER40701, DE-FG03-90ER40569, and DE-FG03-91ER40618), by the Swiss National Foundation (SNF Grant No. 20-118119), and by NSERC Canada (Grant SAPIN 341314-07).

\section{References}

1] E. Komatsu et al., Astrophys. J. Suppl. 180,330 (2009).

[2] G. Steigman and M. S. Turner, Nucl. Phys. B253, p. 375 (1985).

[3] B. W. Lee and S. Weinberg, Phys. Rev. Lett. 39, 165 (1977).

4] S. Weinberg, Phys. Rev. Lett. 48, 1776 (1982)

[5] G. Jungman, M. Kamionkowski and K. Griest, Phys. Rept. 267, 195 (1996).

[6] G. Bertone, D. Hooper and J. Silk, Phys. Rept. 405, 279 (2005).

[7] P. Salucci and A. Borriello, Lect. Notes Phys. 616, 66 (2003).

[8] M. W. Goodman and E. Witten, Phys. Rev. D31, p. 3059 (1985).

[9] R. J. Gaitskell, Ann. Rev. Nucl. Part. Sci. 54, 315 (2004).

[10] T. C. I. Collaboration, Science 327, 1619(March 2010).

[11] D. S. Akerib et al, Phys. Rev. D72, p. 052009 (2005).

[12] Z. Ahmed et al., Phys. Rev. Lett. 102, p. 011301 (2009). 
[13] E. Armengaud et al, arXiv:0912.0805 (2009).

[14] E. Aprile et al., Phys. Rev. C79, p, 045807 (2009).

[15] V. N. Lebedenko et al., Phys. Rev. D80, p. 052010 (2009).

[16] J. R. Ellis, K. A. Olive, Y. Santoso and V. C. Spanos, Phys, Rev. D71, p. 095007 $(2005)$.

[17] L. Roszkowski, R. Ruiz de Austri and R. Trotta, JHEP 07, p. 075 (2007).

[18] R. Bernabei et al., Eur. Phys. J. C56, 333 (2008).

[19] D. Tucker-Smith and N. Weiner, Phys. Rev. D64, p. 043502 (2001).

[20] J. Angle et al, Phys, Rev. D80, p. 115005 (2009).

[21] J. D. Lewin and P. F. Smith, Astroport. Phys. 6, 87 (1996).

[22] S. Yellin, Phys. Rev. D66, p. 032005 (2002).

[23] C. Savage, G. Gelmini, P. Gondolo and K. Freese, JCAP 0904, p. 010 (2009).

[24] R. Bernabei et al., Eur. Phys. J. C53, 205 (2008).

[25] Z. Ahmed, Characterization of supercdms 1-inch ge detectors, in Proceedings of 13th Interational Workshop on Low Temperature Detectors, eds. B. Young, B. Cabrera. and A. Miller, THE THIRTEENTH INTERNATIONAL WORKSHOP ON LOW TEMPERATURE DETECTORSLTD13 1185 (ATP, 2009). 